$\mathrm{p}<0.001)$, a member of $\geq 1$ school sports teams, $(\chi 2=11.6$, $\mathrm{p}=0.001)$, and involvement in a physical fight during the last 30 days $(\chi 2=5.7, p=0.01)$ were some of the risk factors for sustaining an injury. Only $42.3 \%$ had received first aid at school.

Conclusions \& Learning Outcomes Event-based school injury incidence is very high in the study area. Recommendations can be made to adopt safety measures during sports, proper maintenance of the school premises and improvement of first aid facilities.

\section{P4.010 SCOPING REVIEW OF ELOPEMENT BEHAVIOR AMONG CHILDREN WITH AUTISM}

Lise Olsen*, Crystal Shannon, Desiree Thompson, Catie Balehowski. University of British Columbia, Okanagan, Kelowna, Canada

\subsection{6/injuryprev-2021-safety.213}

Background Recreation for children with living with Autism Spectrum Disorder (ASD) is important for improving health and quality of life, however, children with ASD and their families experience many barriers to activity participation. Child elopement behavior is a key barrier that poses a safety concern for parents and is associated with injury occurrence.

Methods We conducted a scoping review, guided by Arksey and O'Malley's framework, to assess the literature on elopement among children with ASD. Search procedures were developed in consultation with university librarian and included searches of five data bases, grey literature, and handsearching. Included articles were those addressing elopement among children 0-19 years with ASD. Abstracts and full text articles were assessed by two reviewers and a third reviewer arbitrated disagreements.

Results A summary of search procedures and key study findings will be presented. Key findings pertain to: nature and patterns of elopement behavior and associated injury outcomes, factors associated with elopement behavior, and preventive intervention approaches and outcomes.

Conclusion This assessment of current literature promotes understanding of elopement behavior among children with ASD, related factors and preventive approaches. This research supports SDG \#10 for reduced inequalities through better understanding of elopement to inform strategies for promoting inclusive and safe recreation participation for children with ASD.

Learning Outcomes Participants will gain understanding of elopement as a safety issue and barrier to equitable recreation participation for children with ASD. Participants will gain knowledge of current research findings about elopement and its prevention.

\section{P4.011 COMMUNITY-LEVEL ESTIMATES OF DEATH DUE TO INJURIES IN MAKWANPUR DISTRICT, NEPAL}

\footnotetext{
${ }^{1}$ Santosh Bhatta*, ${ }^{1}$ Julie Mytton, ${ }^{2}$ Dhruba Adhikari, ${ }^{2}$ Sunil Raja Manandhar, ${ }^{3}$ Elisha Joshi, ${ }^{3}$ Sumiksha Bhatta, ${ }^{4}$ Sunil Kumar Joshi. 'University of the West of England, Bristol, UK; ${ }^{2}$ Mother and Infant Research Activities, Kathmandu, Nepal; ${ }^{3}$ Nepal Injury Research Centre, Kathmandu Medical College Public Limited, Kathmandu, Nepal; ${ }^{4}$ Department of Community Medicine, Kathmandu Medical College Public Limited, Kathmandu, Nepal
}

10.1136/injuryprev-2021-safety.214
Background Availability of injury related mortality data can play a vital role in planning health services for injury prevention and monitoring progress towards Sustainable Development Goal 3.4. In the absence of a robust death registration system, this study explored the feasibility of a model to identify injury deaths occurring outside hospital in rural Nepal.

Methods Data were collected prospectively between February 2019 and January 2020, in two rural municipalities in Makwanpur district. Female Community Health Volunteers notified all deaths in their area to the local Health-Post. Trained data collectors identified the injury-related deaths and invited relatives of the deceased to take part in a structured face-to-face interview.

Results Over one year, 67/451 (14.9\%) deaths were identified as secondary to an injury in the two study areas (injury mortality rate 104/100,000 population). The rate of community injury deaths was higher in males $(128 / 100,000)$ than females (80/ $100,000)$ with a median age at death of 40 years $(S D=20.8$, range 2 to 93). Community injury deaths were most commonly secondary to suicide $(65 / 100,000)$, followed by unintentional injury $(36 / 100,000)$ and assault/violence $(3 / 100,000)$.

Conclusion Large numbers of injury deaths occur outside hospital, and these appear more likely to be due to intentional than unintentional harm. These findings are a valuable additional source of information for engaging stakeholders, developing targeted injury prevention interventions and informing policies.

Learning Outcomes Community deaths secondary to injuries are common and will be missed in hospital-based data collection systems. The mechanism of injury may be different for community compared to hospital cases.

\section{P4.012 A BASIC SURVEY ASCERTAINING DETAILS RELATED TO FALLS ON STAIRS OR ESCALATORS}

Deborah Hilton*. Deborah Hilton Statistics Online [http://sites.google.com/site/ deborahhilton/], ASHWOOD, Australia

\subsection{6/injuryprev-2021-safety.215}

Background The Australian Institute of Health and Welfare state that falls account for $41 \%$ of all hospitalised injuries. In Melbourne City, at various train stations there are extremely long escalators and alarmingly one sign previously displayed states there are approximately 2-3 falls/week.

Methods A general convenience survey [ $n=125]$ was distributed to ascertain the percentage of people who've fallen down stairs and/or escalators. Additional questions related to rain, high risk footwear, poor lighting and how many falls they have had. Following on from that regardless of whether or not they'd had a fall, survey participants were asked about fear of falling and/or whether they felt uncomfortable around crowds on stairs or escalators.

Results 82 survey forms were returned. 29\% of people had fallen, with $16 \%$ of those people indicating rain, $16 \%$ in either high heels or thongs, while $25 \%$ indicated poor visibility. of those whom had fallen the estimate of the number of falls down stairs or escalators ranged from 1-20, with the average being 3 falls.

$39 \%$ of respondents irrespective of whether or not they had fallen had a fear of falling, while $43 \%$ felt uncomfortable around crowds. The age groups were 33\% [20-29 yrs], 8\% 